Article

\title{
Surprise and Uncertainty-Framing Regional Geohazards in the Theory of Complexity
}

\author{
Beate M. W. Ratter \\ Institute of Geography, University of Hamburg, Bundesstrasse 55, Hamburg 20146, Germany; \\ E-Mail: ratter@geowiss.uni-hamburg.de; Tel.: +49-40-42838-5225; Fax: +49-40-42838-4981
}

Received: 20 November 2012; in revised form: 17 December 2012 / Accepted: 26 December 2012 /

Published: 4 January 2013

\begin{abstract}
The paper analyzes the concepts of uncertainty and surprise as key variables of a socio-ecological system's behavior in the context of the theory of complexity. Experiences from the past have shown that living with uncertainty is part of our daily life and surprises are only surprising because our perspective of system trajectories is basically linear and non-dynamic. The future of humanity is dependent on the understanding of the system's behavior and needs a change in perspective of linearity to non-linearity and from the planning imperative to a management hedging uncertainty and surprise. In the context of humanity's future, the theory of complexity offers a new perspective on system trajectories and their understanding of surprises and uncertainty. There is a need for a Gestaltwechsel - a change in perception — which helps to see things differently and fosters the search for new answers to emerging questions at the human-nature interface. Drawing on the case study of hazard management the paper will explain the necessity of analysis system's behavior and the taking into account of multi-agent behavior on the micro level which led to emergent behavior on the macro-level of the system. Regional geohazards are explained as the regional impact of an uncontrolled risk based on a state of a natural feature that has a direct impact on a regional population being affected by the appearance of a hazard and its development into damage. By acting in space, time and connectivity, people construct hazardscapes and change risk into regional geohazards. This concept shows relevance for future mitigation and adaptation measures. The theory of complexity can help in engendering the necessary shift in perspective. What is non-linear dynamic thinking as suggested by the theory of complexity? Why is the consideration of the system's behavior crucial and not just the number of system's elements? What is the role of agents in these systems? In addition, there are practical implications too: What does this shift in perspective mean for future hazard management and the future of humanity?
\end{abstract}


Keywords: regional geohazards; complexity; surprise; uncertainty; hazardscape; hazard management

\section{"There are known knowns.}

These are things we know that we know.

There are known unknowns.

That is to say, there are things that we know we don't know.

But there are also unknown unknowns.

There are things we don't know we don't know."

Former US Defense Secretary Donald Rumsfeld, 12 February 2002 in a press briefing

\section{Introduction}

On 12 January 2010, a serious earthquake measuring 7.0 on the Richter scale destroyed the southern part of Haiti including the capital, Port-au-Prince. An estimated 300,000 people died, another 300,000 were injured and up to 1.2 million were left homeless. Did this come as a surprise? Not to those familiar with the geology and plate tectonics of the northern Caribbean. Statistically speaking, and looking back over history, an earthquake had long been overdue. After being shaken by serious earthquakes in 1618, 1673, 1684, 1761, 1770, 1860 and 1946 the question was not if but when. When would the Haitian people once again be exposed to this type of natural disaster?

From geology's perspective, Haiti can be described as a high-risk space since earthquakes as well as hurricanes and landslides form part of the natural system's trajectory. The island is a geohazardous area. However, the physical world, along with the biological and social worlds that populate it, is only inadequately described by this. Each of these worlds represents highly contingent, emergent complex systems, which interact at different temporal and spatial scales. Understanding the characteristics of these systems requires an expansion of our idea of science, recognizing that good scientific practice reaches beyond the Newtonian paradigm characterized by a stable world of laws that can be described by linear relations of cause and effect. The complexity paradigm particularly applies to the role of context in shaping natural and social phenomena, which is essential for understanding the complex behavior of non-linear dynamic systems. Surprise and uncertainty, both of which are intrinsic to complex system behavior, represent mental constructs, and although they are linked to natural conditions, they are equally embedded in the experiences and cognitive processes of a particular society. The frequency and intensity of a disaster is a constituting element of social memory and shapes how society conceives of the space it inhabits.

In this paper the concepts of uncertainty and surprise is analyzed in the context of the theory of complexity. Not that general forces of vulnerability-poverty, politics or economics-have no influence in the handling of natural and other disasters, but the focus of this paper simply tries to move the focus from current scientific understanding towards an in-depth analysis of the system's behavior. The theory of complexity offers a new perspective on system trajectories and their understanding for 
the future of humanity. I intend to show that living with uncertainty is part of our daily life and that surprises are only surprising because our perspective on system trajectories is basically linear and non-dynamic. We need a Gestaltwechsel-a change in perception-which helps us to see things differently and fosters the search for new answers to emerging questions at the human-nature interface. "It means that conditions often relegated to the status of 'accidents' or 'boundary conditions' in the old paradigm must be elevated to the subject of scientific study in their own right. Historical contingency conspires with episodes of randomness to create the actual forms and behaviors that populate the social world" ([1], p. 4).

The theory of complexity can help in engendering this necessary shift in perspective. What is non-linear, dynamic thinking as suggested by the theory of complexity? Why is it that the behavior of a system is crucial and not just the number of system elements? What is the role of agents in these systems? There are practical implications too: What does this shift in perspective mean for hazard management?

\section{The Theory of Complexity}

The term "complex" is used in many different ways and encompasses a great variety of phenomena. In everyday language "complex" has become another way of saying "complicated", although the following will show that complex is not the superlative of complicated [2]. Complexity, complex and complex systems are also widely used within the scientific community. Here too, uncritical use of the terms and the lack of a distinct and unambiguous definition carry the danger of turning them into empty phrases.

The theory of complexity is a clear-cut concept. Rooted in chaos theory, it provides a perspective on system behavior, portraying them as non-predictable and non-mechanistic, full of surprises and constantly evolving. Theories on "complex systems" attempt to explain how relationships between system components give rise to patterns of collective behavior that define the properties of the "whole system", which interacts and forms relationships with its environment. Complexity theory can provide a constructive and innovative perspective on non-linear dynamic system behavior.

Complexity theory holds that the trajectories of non-linear dynamic systems are determined by the fact that single agent interaction at the micro level can lead to emergence at a macro level. These trajectories are full of jumps and bifurcation points: Forks in the road where the system could take an entirely different path but does not necessarily do so. Trajectories also have no clear cause-and-effects. An essential characteristic of non-linear dynamic systems is thus that surprise forms an inherent feature of their development.

\subsection{Structural Versus Behavioral Complexity}

The unpredictable outcome of a system's development is not only down to the interaction of many different individual elements, which may give rise to complex structures. A fundamental aspect in the complexity view of systems is the distinction between structural and behavioral complexity [3].

Most of the systems comprising an interaction of humans and nature are composed of a wide range of interacting elements. Systems are said to be structurally complex when they consist of many different elements and interactions. Behavioral complexity, in contrast, arises from the processes and 
relations between the system elements. Contrary to other interpretations of complexity, behavioral complexity cannot be reduced, but must be accepted as a fact. At the same time, behavioral complexity does not automatically imply a complicated structure. A system that exhibits behavioral complexity can consist of simple or complicated structures [4]. This point can be illustrated by considering the behavioral complexity of a double pendulum. A double pendulum is a very simple physical system consisting of a pendulum A attached to another pendulum B. Although each pendulum is a simple mechanical system, the joined double pendulum AB exhibits complex behavior [5] (see Figure 1).

Figure 1. Double pendulum with canvas traces [5].

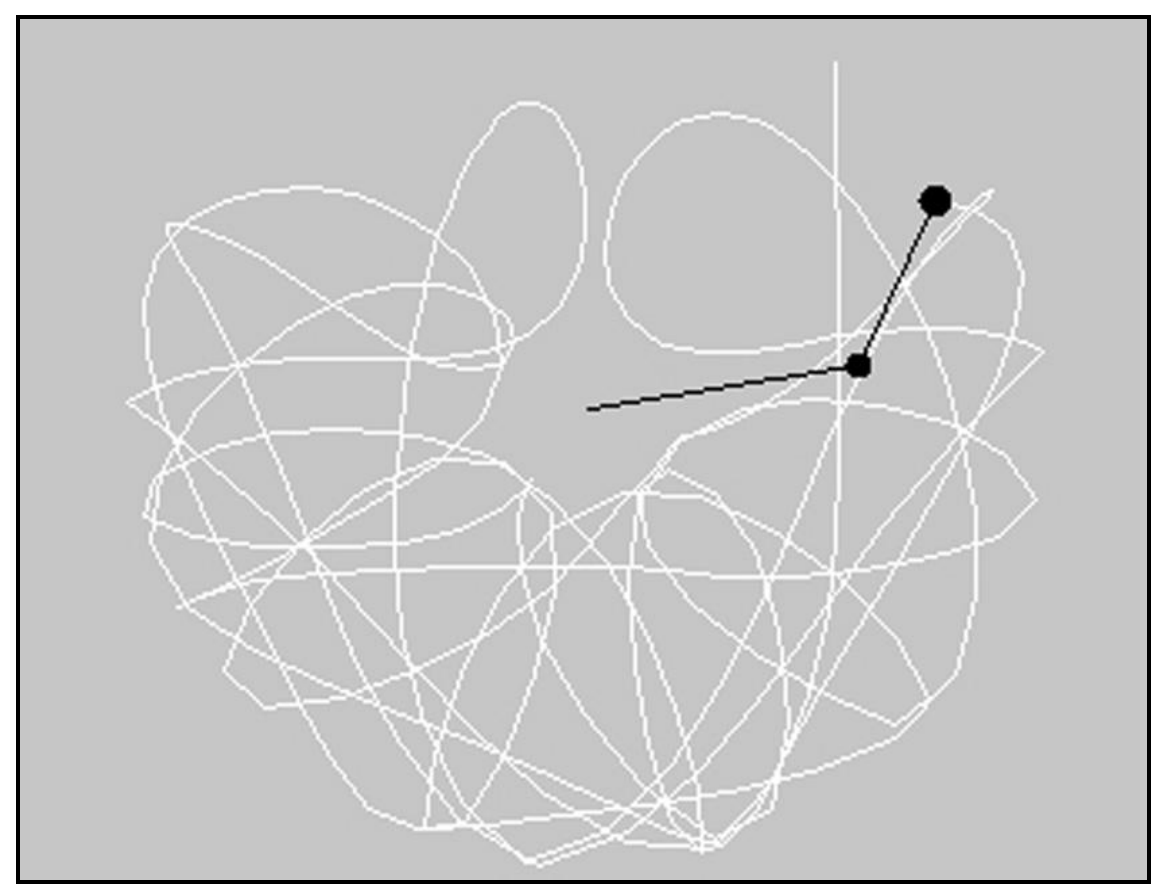

The double pendulum is an example of applying the theory of complexity to system behavior rather than structure. If even simple systems can show complex behavior, the same must be true for structurally complex systems, which would thus be structurally and behaviorally complex. The fundamental question though is this: How can something complex arise from the simple? Clearly, behavior can not be understood by only referring to the properties of individual elements. Rather, it is the iterative interactions of the system elements that count. In the case of structurally complex systems such as the weather or the stock exchange, complex behavior is determined by the interaction of many of the system's constituting elements and/or agents. In such dynamic systems, the elements interact in a non-linear way, which can result in the emergence of complicated and above all counterintuitive behavior. A complex system can display surprising properties when elementary sub-processes are coupled together to form a larger system ([6], p. 40), and "the interactions between the parts are far more fundamental than the parts themselves" ([7], cited in [8], p. 4). Local micro-level activities of agents therefore take on global significance in that they influenced macro-level system behavior ([4], p. 57ff; [9]). 


\subsection{Agents Not Actors}

As stated above, the interaction of the system's constitutive elements at the micro level is decisive for the emergence of new structures on the macro-level. These constitutive elements are termed agents. Individual agents act based on restricted information and to a large extent with self interest; nevertheless they are key in shaping the trajectory of the larger system.

Traffic congestion is a simple but useful example to illustrate the emergence of new structures at the macro level of a social system. We all get stuck in traffic jams fairly regularly-but do we ask ourselves why this happens? Traffic congestion can emerge without a specific cause merely as a result of drivers following simple rules. If we assume these rules to be to drive at a certain speed, to not crash into the car in front; to slow down if there is a car close ahead, and to speed up if not, traffic jams start from small seeds, emerging randomly so to speak from the original positioning of the cars and their random original speeds. "When cars cluster together, they will move more slowly, causing the cars behind them to slow down, resulting in a traffic jam. Even though all cars are moving forward, the traffic jams tend to move backwards. The behavior of the group is different from the behavior of the individuals that make up the group" [10]. The sum of agent behavior leads to entirely new system behavior (see Figure 2), just as a traffic jam emerges out of the behavior of single agents.

The theory of complexity demands that a clear distinction is drawn between agents and actors. 'Actor' is the more general and superordinate concept in which agents can be described as a particular type of actor. Actors have also been described as creating spatial clusters of quasi symbiotic unities or combating complexes [4,6]. An actor-based perspective focuses on intentional action and influencing the system's behavior. An agent-based perspective focuses on the emergence of new macro-level phenomena as the result of agent interaction at the micro level.

Figure 2. Abstract illustration of emergence in complex systems ([11], p. 25).

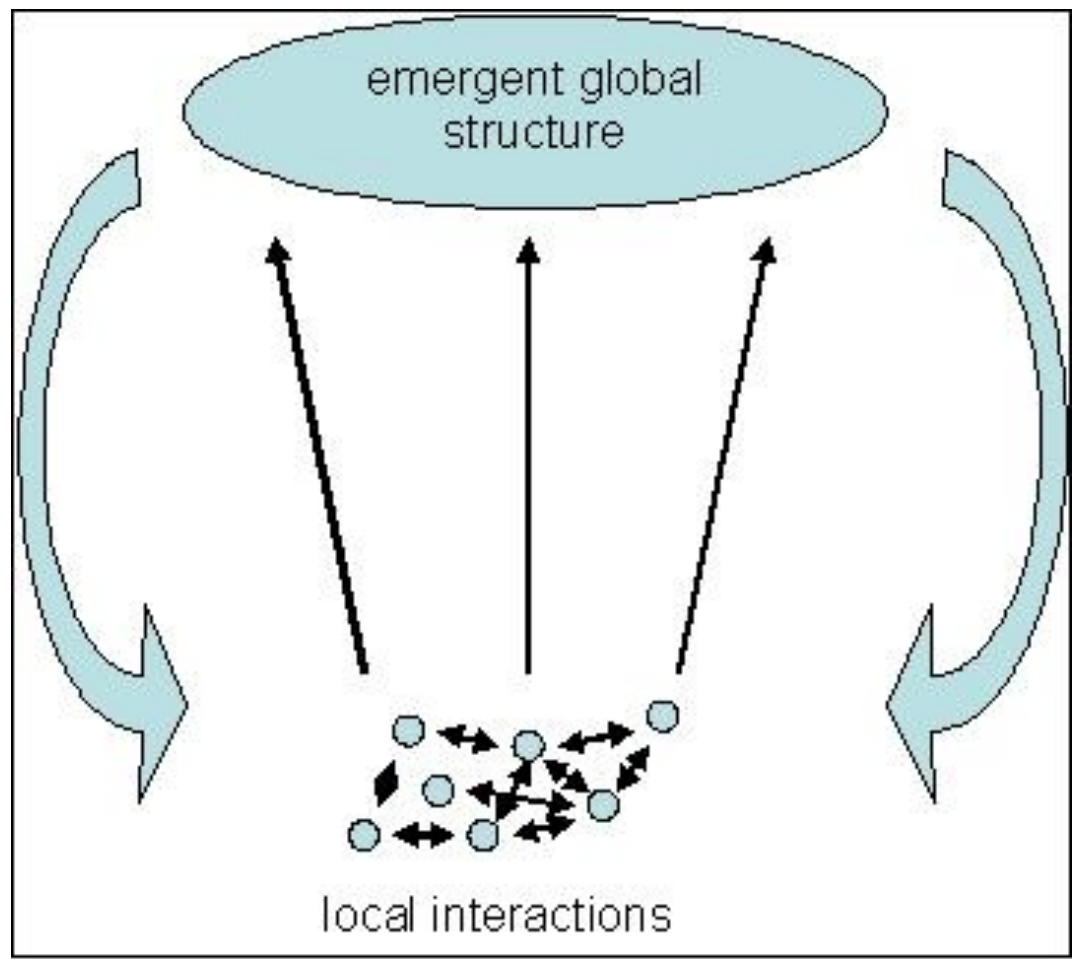


Contrary to other types of actors, an agent has limited or no knowledge of the system as a whole. They therefore represent a system unit or element that acts locally and cannot directly influence the overall system. But although agents are restricted in their actions, these very actions can still change the system through iteration and repetition, even if the impacts of an agents' activities beyond the local scale are indirect and do require some form of mediation. There are obvious parallels to the field of secret agents: those engaged in the cloak-and-dagger games of the former superpowers had similarly limited knowledge of the system as a whole, knowledge in other words that was restricted to their own respective areas of operation.

Behavioral complexity is therefore a crucial parameter in that it considers the qualities and properties of a system as well as the relationships and processes between its constituent elements. Non-linear behavior, and by implication behavioral complexity, are irreducible, with the behavior of the system strongly influenced by the system's history. Nonlinearity can drastically affect predictability. This implies that structural reorganization has no single cause, but arises from micro-scale inter-dependencies that act together to cause the spontaneous appearance and disappearance of order and structure. Throughout, emphasis is on the 'becoming', with complex meaning birth, evolvement and emergence, all of which can lead to profound qualitative transformations [2,12]. This understanding of system's behavior is not new, since Aristotle already stated: "the whole is more than the sum of its parts". What is important however for humanities futures, that we recognize the system's non-linear, dynamic behavior as integral part of our analysis and perspective.

\section{Uncertainty and Surprise — Two Concepts in Non-Linear Dynamic Systems}

The theory of complexity informs us that jumps are intrinsic in non-linear, dynamic systems. We have to live with surprise and the uncertainty of the outcome of a system's trajectory. But what is surprise? And what is uncertainty?

Surprise can be defined as a brief emotional state that is the result of experiencing an unexpected event. Surprise can have validity in that it can be positive, neutral or negative, and particularly powerful or long-lasting surprises can be experienced as shocking. Surprise is a relative, subjective entity because it is linked to the expectations of an observer, be they a single synapse, neuronal circuit, organism, or computer device. In mathematics, the same data may carry different amounts of surprise to different observers, or even to the same observer at different times. However, more importantly perhaps, surprise is inextricably linked to uncertainty, which can arise from intrinsic stochasticity, missing information, or limited computing resources. A world that is purely deterministic and predictable in real-time contains no surprises.

Uncertainty is employed by a range of scientific disciplines, each of which has their own definitions and understanding of the term. It also features in many applied contexts, such as the prediction of future events or the reliability of physical measurements. Defined as a human experience, uncertainty is a relative and subjective entity, a social construct, which is affected by the individual and collective processes that lead us to anticipate the future. Rayner [13] describes uncertainty as a function of how individuals and groups perceive their place in the world and the things that threaten it (compare "subjective uncertainty" according to [14]). As an experience, uncertainty manifests itself as an 
emotional state: Permanent changes in living conditions, for example, can cause a state of fear, helplessness or loss of orientation ([15], p. 25).

Beyond the experiential, uncertainty can also refer to a situation where possible events per se are known, but where it is impossible to determine the likelihood or timing of these events ([15], p. 22). This is certainly true for the case of an earthquake. In this context, uncertainty can be defined as the lack of certainty, or a state of limited knowledge, where it is impossible to exactly describe an existing state or future outcome, what Donald Rumsfeld, in the quotation that began this article, would call a 'known unknown'. D. Lindley, a statistician, states that "you are uncertain, to varying degrees, about everything in the future; much of the past is hidden from you; and there is a lot of the present about which you do not have full information. Uncertainty is everywhere and you cannot escape from it" ([16], p. xi).

N. Knight [17], a planner, identifies three types of uncertainty, each of which has different origins. The first is uncertainty conditioned by the environment (environmental uncertainty), the second uncertainty that springs from variable evaluation or assessment (value uncertainty), and the third uncertainty that originates from coordinating processes (related choice). The first is the result of insufficient information about the environment. In this context, environment is understood as a system that is subject to external influences outside human control. The second is related to problems arising from multiple value contexts, in particular the difficulty of assessing future preferences in the context of changing individual and social norms. In the third case, uncertainty results from the fact that the consequences of any action taken by an individual or institution also depend on other people's action. Coordination processes are therefore the source of the third type of uncertainty.

An important aspect is that "... uncertainty itself has no ethical quality - it is an inherent attribute of a situation. However, in a potentially dangerous situation uncertainty can trigger ethically adjusted behavior that aims to avoid dangers and diminishes risks" ([14], p. 892). Here, the authors distinguish between objective and subjective uncertainty as two main branches, where the first contains epistemological and ontological uncertainties, and the second moral and rule uncertainties. While the former is caused by gaps in knowledge that can be closed by research, the latter is characterized by the inability to apply appropriate moral rules. Ontological uncertainty is caused by the stochastic features of a situation, which will usually involve complex technical, biological and/or social systems. Since complex systems are characterized by nonlinear behavior, it is - at least in a non-lab situationimpossible to resolve uncertainties by deterministic reasoning and/or research ([18] cited in [14]). "The effects of interfering with financial markets or ecosystems, for example, are largely unpredictable; nevertheless, past experience and probabilistic reasoning at least provide some guidance on how such complex systems will react." ([14], p. 893).

In consequence, uncertainty may either result from a lack of knowledge of obtainable facts (the known and unknown knowns), or the unpredictability of future emergences (the unknown unknowns). As established above, uncertainty is an integral part of the trajectory in complex, non-linear systems: The future will always be uncertain because the interaction of agents at the micro level may result in unpredictable emergences at the macro-level. 


\section{Uncertainty, Not Risk}

Uncertainty is not the same as risk or probability. As early as 1921 the economist Frank Knight stated that "uncertainty must be taken in a sense radically distinct from the familiar notion of risk, from which it has never been properly separated. The essential fact is that 'risk' means in some cases a quantity susceptible of measurement, while at other times it is something distinctly not of this character; and there are far-reaching and crucial differences in the bearings of the phenomena depending on which of the two is really present and operating. It will appear that a measurable uncertainty, or 'risk' proper, as we shall use the term, is so far different from an immeasurable one that it is not in effect an uncertainty at all" ([19], p. 20).

Risk can generally be defined as a situation in which the behavior of a system is widely known and estimates are made as to the probability that a certain event or chain of event will occur. Risks in this context can be reduced to a simple formula: Risk $=$ damage $\times$ probability. "An example would be estimating the risk of a specified magnitude of flood based on a long-term record of water flow in a river system. The risk could be calculated with long-term information. There would always be a possibility of error, but there could be considerable confidence in the estimate." ([20], p. 3) Uncertainty, in contrast, refers to situations where we do not know how the system might react. While the risk of rain is calculable based on weather simulation and forecast, the development of the stock exchange is not. Although we can estimate what might happen, no objective facts or parameters exist which could allow us to calculate likelihood ([15], p. 23; [21]). Risk can thus be calculated, uncertainty cannot.

\subsection{How to Cope with Uncertainty?}

It is a human desire to be as safe and certain as possible. We want to foresee the future as much as possible and are keen to prepare for hazards and surprises technically and socially. Despite the many scientific efforts dedicated to planning and forecasting, simulating, modeling and predicting, as well as the whole range of individual insurances and our hedging against them, surprise and uncertainty continue to determine our world. It also seems that the more scientists and planners model the future, the more we begin to anticipate unprecedented surprises, the greater our uncertainty. So what is going wrong?

After more than two centuries of studying equilibria, a shift in reflection is required, focusing on the emergence of structures and the unfolding of patterns in natural and social systems. Arthur [22] states that "small events (the mutations of history) are often averaged away, but once in a while they become all-important in tilting parts ... into new structures and patterns that are then preserved" ([22], pp. 11, 12). Complex systems often contain feedback mechanisms that result in the amplification or dampening down of the outcomes of non-linear chaotic behavior. These are conditions where causal explanations by addition will fail.

Both concepts of surprise and uncertainty are based on human agents, which are constituent parts of the social system. Human agents are embedded in their regional culture. They live in a cultural environment, have historical and cognitive knowledge, and experience emotions. Agents perceive their environment individually, physically and mentally constructing their environment according to their 
interests, beliefs and needs. Society is constructed by agent interaction. In addition, society itself has a history, its own relationship to the environment and its own prior experience with uncertainties and surprises. These include surprises that go far back in history, may have paled in significance in the collective memory of society and will at the same time influence the trajectory of humanities future. The main point is that society's development is contingent, and that agents are embedded in this society and their respective spatial environment. The relevance of this is demonstrated below with two examples from the Caribbean: the earthquake in Haiti and hurricanes in the Bahamas.

\subsection{Risk Space or Hazardscape-A Difference}

Risk is the calculable probability that an extreme event will occur. This definition of risk also refers to the possible damage a disaster may cause; hence, risk is a function of both magnitude and probability of an event (cf. [23], p. 50). Natural disasters are prime examples of such events that take place at a local, regional or supraregional scale. Their spatial dimension is obvious. An earthquake, for example, is linked to active plate tectonics, its occurrence the result of regional instability and tectonic activity. A volcanic eruption is linked to a specific spatial feature (a physical volcano), its ecological and social impacts affecting its slopes and the surrounding areas. At the same time, the precise spatial impact of such events is unpredictable. A tropical cyclone develops over warm water masses and follows a likely corridor, but its actual trajectory depends on many influences such as topography, pressure differences, or wind directions. Although its pathway per se is contingent, it is hardly possible to predict just which of the many potentially affected areas it will hit.

A hazard is defined as a potential threat to humans and their belongings caused by an extreme event. As such, a hazard is vague in that it only shows the potential of an occurrence in a certain predisposed area. Like uncertainty, and unlike risks, hazards are non-calculable. Hazards are threats that arise from the unpredictability of a system's behavior and the interaction of the system's agents. Therefore, hazards are emotionally and cognitively charged constructs.

Space is constructed both consciously and unconsciously in the cultural context of a society and based on perceptions of nature and the environment [24]. Mental and material constructs of space are linked to each other by human practice. Scape, in contrast, is a principle that makes it possible to consider part of space as an individual unit. The significance of landscape, for example, can be clustered around the fundamental components of form (the physical and tangible aspects of a landscape), practices (the activities associated with a landscape) and relationships (the meanings generated between people and their surroundings) [25]. 'Scaping', or the turning of objective space into subjective scape, is an active, iterative, creative process where mental and material spaces repeatedly constitute one another. This is transported by the German term 'Landschaft', whose etymology carries the creative act of delineating and also shaping a distinct unit of land [26].

If risk refers to calculable risk (e.g., of a natural disaster) and hazard to a general threat, the same is true for the respective areas affected. Risk space, meant here in the sense of space potentially at risk, can be determined by calculating the intensity and outcome of an event. However, in many situations, such a calculation is impossible, and even if it were, we would probably not do the math. This is because we get used to a hazard, become hardened to it or simply stop thinking of the potential threat. A hazardscape is therefore a space that is confronted with an undefined yet existing threat, which is 
neither predictable nor calculable. Hazardscape in this sense is understood as an emotionally charged landscape, which is constructed based on perceived threats and fears or fatalism. Hazardscapes are social, visual and affective entities that are made and unmade in a particular cultural and societal context.

A geohazard is defined as a "geological state, which represents or has the potential to develop further into a situation leading to damage or uncontrolled risk. Geohazards are found in all parts of the earth and are always related to geological conditions and geological processes, either recent or past." (International Centre for Geohazards, Oslo). A regional geohazard can be defined as the regional impact of an uncontrolled risk arising from a natural feature with direct impact on the regional population; the population will be affected by the appearance and the development of a hazard into damage. As a rule, and in a long-term process based on past experience, the regional population has developed adaptation strategies to mitigate the impact of such potential damage (i.e., dykes along low lying coastal areas, hurricane or earthquake safe housing, stilt houses in flood prone areas, etc.). However, unprecedented effects can result in new dimensions of disaster, exposing the regional population to the impacts of a regional geohazard despite the presence of traditional adaptation and mitigation measures. Studying regional geohazards therefore requires a combined approach, considering not only the potential natural threats but also society's preparedness for dealing with them. The ability to deal with hazards depends on information, experience, threat perception and governance structures. Handling regional geohazards must be based on an integrated perspective combining natural and social sciences and taking account of the uncertainties and surprises of non-linear, dynamic systems.

Whereas risk space is confronted with the physical risk in a certain area, hazardscape arises from constructs of uncertainty, which are fed by memories, traditions, as well as mental and physical processes. A hazardscape manifests itself in physical space in Urry's sense that "the effects of humans are subtly and irreversibly woven into the very evolution of landscape" ([8], p. 6). Based on the concept first defined by Corson [27] in a technical context ${ }^{1}$ and transformed by Cutter, Mitchell and Scott in 2000 in their study of place vulnerability [28], hazardscape can thus be defined as ecological hazards and risks that build through a constant, implicit and intricate relationship between human beings and the environment in a particular spatio-temporal context [29].

\section{Contingency in a Space-Time Continuum-The Haiti Example}

The above has revealed that the evolution of any complex system is defined by emergent behavior and jumps, making surprises a constituent part of the system's trajectory. "The 'normal' state of nature is thus not one of balance and repose; the normal state is to be recovering from the last disaster." ([8], p. 5) A complexity-based perspective can thus enable us to break with the common dualistic thinking in systems or system failures [30]. The destruction of Port-au-Prince on 12 January 2010 in a 7.5 magnitude earthquake is a notable example.

Haiti is situated on the northern margin of the Caribbean plate, which is pushing east, moving against the North American plate, which is pushing west. Plates not only grind past each other, but are

1 .... hazardscape is the spatial distribution and attitudes of human engineered facilities... that contain or emit substances harmful to humans and environment ([27], p. 57). 
also compressed in some areas on account of the irregular shape of the plate boundary. Plate motion is about $20 \mathrm{~mm} /$ year. Tectonic movement creates two east-westerly fault zones, the Septentrional fault that runs along the northern side of Hispaniola, and the Enriquillo-Plantain-Garden Fault Zone (EPGFZ) that extends along its southern side (see Figure 3). US Geological Survey [31] experts estimate that the EPGFZ fault is to blame for the historical earthquakes in previous centuries. The last large earthquake along the northern fault occurred in 1946, which had a magnitude of 8.0. Research published in 2008 warned that tectonic tension had been building along the Enriquillo-PlantainGarden-fault since 1751, when the last large earthquake in the region struck the southern part of the Dominican Republic, and forecast a magnitude of 7.2 for an earthquake that would eventually result in release of this tension [32].

Figure 3. Haiti-Enriquillo-Plantain-Garden-Fault Zone [33].

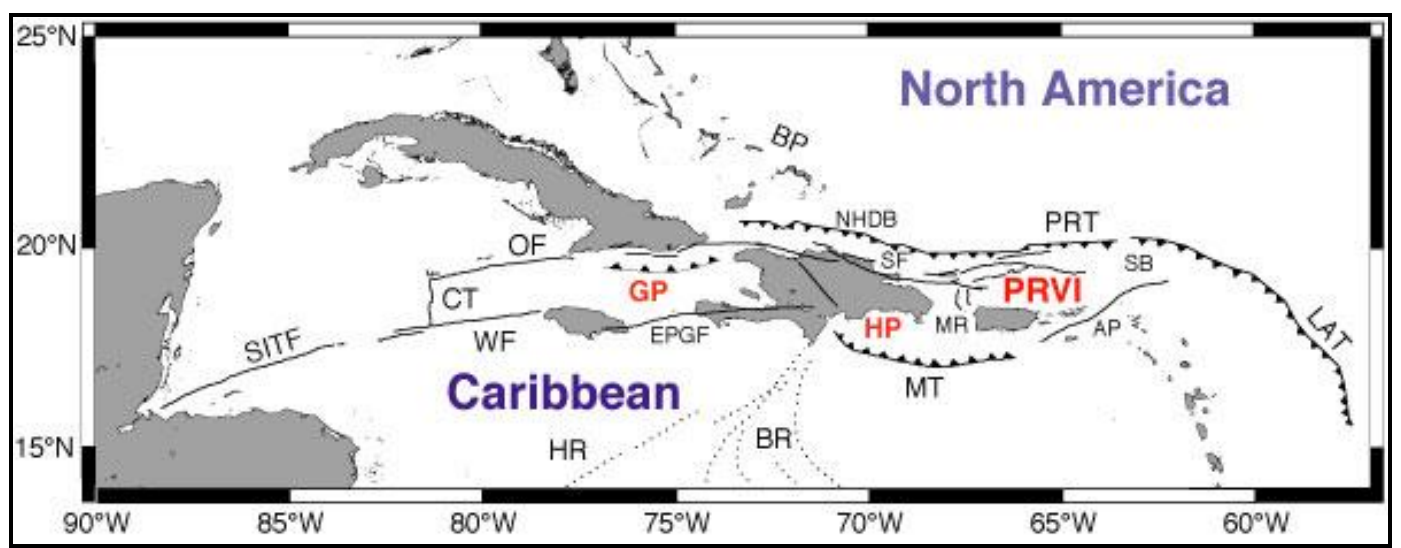

Map of northern Caribbean plate boundary showing microplates and structures; AP: Anegada Passage; BP: Bahamas Platform; BR: Beata Ridge; CT: Cayman Trough Spreading Center; EPGF: Enriquillo-Plantain Garden Fault; GP: Gonvave Platelet; HP: Hispaniola Platelet; HR: Hess Rise; LAT: Lesser Antilles Trench; MR: Mona Rift; MT: Muertos Trough; PRVI: Puerto Rico-Virgin Islands block; SB: Sombrero Basin; SITF: Swan Islands Transform Fault; SF: Septentrional Fault; WF: Walton Fault.

The location of the epicenter of the earthquake of January 2010 was $25 \mathrm{~km}$ southwest of the capital of Port-au-Prince. It destroyed the presidential palace, Port-au-Prince Cathedral, the Anglican Holy Trinity cathedral, a former children's hospital, the port and the airport. The slums of the city, with over a million inhabitants, were particularly badly affected by landslides. Chaos reigned in the immediate aftermath of the earthquake, with several factors conspiring to hamper rescue efforts. The earthquake struck an hour before dark, taking out both power and telephone lines. Haiti had no contingency plan for such a disaster, and the medical infrastructure that existed was either destroyed or proved unable to cope with overwhelming demand. The general scientific analysis is focusing on the vulnerability of the Haitian society, the manifest failures in society, politics and economics. No question that these failures were unaided by the fact that the Haitian society is especially vulnerable to such a disaster and this should not be ignored. However, there is a need in complementing this perspective by shifting the focus on non-linear surprises and uncertainties.

Although exemplifying regional geohazards-showing historical experience and a specific geological position along two tectonic faults - the large earthquake of January 2010 hit the island as a surprise. Haiti is a risk space and an earthquake should have been expected. However, because the last 
large earthquake occurred 40 years ago, the population had begun to feel 'safe'. A mental construct had arisen of the area as a place safe from earthquakes. As a result, building materials or building codes did not take into account the potential occurrence of earthquakes. It was the rigid reinforced concrete structures that were first to collapse in the January 2010 event. Not only mentally, over decades Haiti was constructed as a hazardscape. The regional geohazard became manifest.

\section{Contextuality of Culturally Embedded Agents-The Bahamas Example}

The perception of hazards is embedded in regional culture; within each culture, it finds specific expressions. The second regional example draws from the Bahamas experience. Hurricanes are a regular occurrence in the Caribbean (see Figure 4). In the English-speaking part hurricane season is described in a well-known nursery rhyme that is based on general experience:

June-too soon.
July—stand by!

August—look out you must.

September-remember.

October, all over.

A hurricane's (spatial) impact is difficult to predict just as that of an earthquake because both its strength and path are uncertain. A hurricane can grow in strength or weaken, dissolve, gain speed, stay on its course or suddenly change track. Everything depends on small influencing factors that cannot be predicted beforehand. Despite careful monitoring of weather conditions, hurricane forecasts can only be made a few hours in advance. Contingency plans for this natural disaster need to take this into account.

For the population, hurricanes are part and parcel of a Caribbean summer - natural events that form part of everyday life. On small islands, uncertainty can breed certain fatalism; after all, one can never be sure whether the next hurricane might destroy all one's worldly goods. Overhead power lines are typical for Caribbean islands but they are unsuitable for countries frequently hit by storms. Unfortunately, putting the power lines under ground is more effort and expense than simply re-erecting the fallen utility poles every year. The leaky, vulnerable wooden houses are another problem. These are prevalent throughout the Caribbean and extremely susceptible to storm damage. The massive concrete hotels usually survive without damage and sometimes even serve as hurricane shelters for the local population. Social vulnerability and resilience thus become central issues in the hazard debate. However, it also depends on the social and cultural construction of hazardscape the people inhabit.

Absolute wind speeds or intensities only partly determine the impacts of hurricanes on Caribbean societies. Coping with natural disasters implies an ongoing process of preparation, taking precautionary measures, getting ready and, wherever possible, using technology in the protection against extreme events (e.g., flood defense, special architecture and construction of hurricane-proof houses, etc.) Importantly, pre-disaster preparation is only one element in the iterative process of disaster management, which apart from preparation also comprises immediate disaster relief and post-disaster relief management. Integrative Hazard Management also means preparing society for the potential impacts of a natural disaster. Every year, Caribbean island states go to great lengths to 
prepare their population for the hurricane season. Radio broadcasts, newspaper ads, posters, exhibitions, special teaching units at school, and special mugs are used to attempt to firmly lodge the idea of risk in people's heads. If hurricanes are merely considered a potential risk rather than an inevitable one, acceptance of risk must come as a necessary first step before adaptation or mitigation.

Figure 4. Hurricane trajectories on the Bahamas.

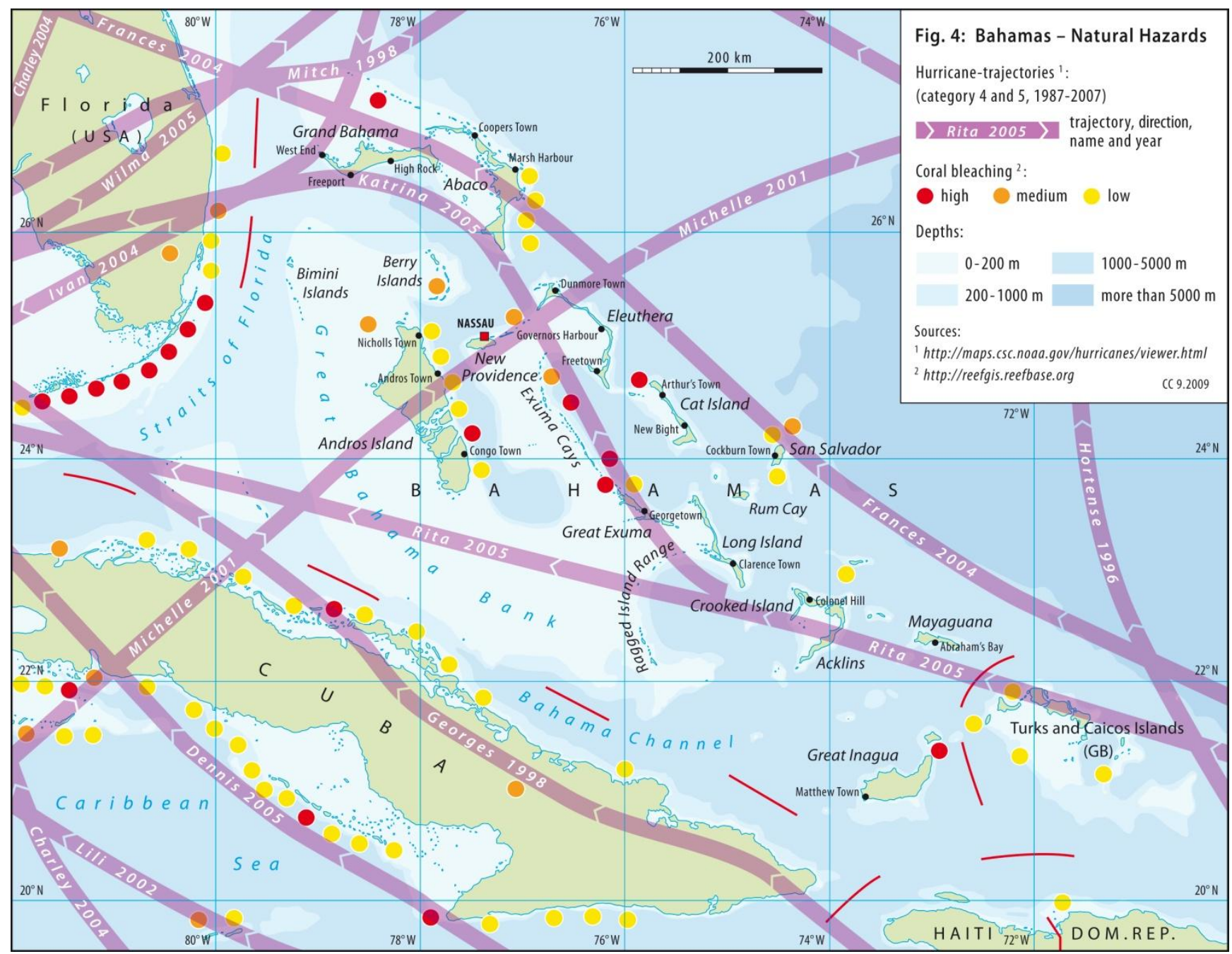

\section{Risk Awareness and Governance}

With respect to hurricanes, Caribbean islands are clearly risk spaces and show the critically of regional geohazards. However, although hurricanes pose a constant threat throughout the summer months, only those hurricanes that are important to the community actually strike. In the Bahamas, a gateway of storms to the USA, the saying goes that "only the hurricane that hits you matters!" The Bahamas were fortunate in that no severe storms were encountered in the 1970s and 1980s. Major change did not take place until 1992 when Hurricane Andrew had a severe impact on the islands. Institutional arrangements were altered, the National Emergency Management Agency (NEMA) was established and governmental preparedness programs were put in place. However, did this event really change hurricane awareness in the Bahamas? 
After a long period of relative calm, Hurricane Andrew led to four casualties in the Bahamas. Overall, damage was estimated at \$250 million US\$. The island of Eleuthera was affected worst, but subsequent severe storms also caused damage on different islands [34]. In 2008 Hurricane Ike, a category 5 storm, hit Great Inagua with its population of 950, but spared the much larger population of New Providence. For any kind of awareness-raising, knowing where the hurricane will hit and how much impact it might cause nationally is therefore essential. Awareness in turn depends on previous experience, says Captain Stephen Russell, Coordinator of the National Emergency Management Agency (NEMA): "We have a bad habit on the Bahamas, people only act when they feel the breeze!"

In the nation's capital Nassau on New Providence, people feel comfortable. New Providence has not experienced a hurricane for a long time and feels safe because it is protected by the other islands. “This is a false bubble!" says Diana Turnquist, former Disaster Officer of the Bahamas Red Cross. "We are the least prepared. Because of lack of historic experiences in New Providence people have no hazard consciousness. They need a wake up call."

For the Bahamas the chances of a hurricane hitting and the respective damage this might cause represent a calculable risk. However, for the single island's communities exposure to a major hurricane represents a hazard, as do the ensuing flooding events, which cause additional damage to houses and infrastructure. New Providence with the capital Nassau exhibits localized thinking, only considering New Providence instead of the wider island environment. Ninety percent of the population lives on the island, most of the nation's economy and infrastructure are located here, and they have never really had a shock. The hazardscape is explained by an anonymous interviewee in Nassau in October 2011: "It is just our culture, a hand to mouth symptom. I do not care about tomorrow, I live for today." Against the mindset that only the hurricane that hits really counts, a mentality of pre-planning or proactive management is absent in this socio-cultural environment. This can become a problem in the overall process of hazard management, where preventative action and preparation are important elements.

\section{The Theory of Complexity and its Contribution Hazard Management}

The case of Haiti demonstrates that surprise depends on the perspective taken and the conceptual framework applied. Since the mid 1980s leading hazard scholars have referred to hazards as open and complex systems for which a multitude of elements is just as characteristic as cyclic and instable transformation processes [15,23,35-43]. They call for an integrative approach in hazard research and management and specifically refer to the theory of complexity in their deliberations and research. However, integrative approaches are still not being implemented. Although non-linearity, emergence and feedback are repeatedly mentioned in the literature the Gestaltwechsel from complicated to complex system behavior has yet to be broadly acknowledged and its implications recognized in hazard management. People prefer to think in linear sequences, and reliance on linear development abounds in everyday life and in hazard management. Why is that?

There is a need to acknowledge that interaction between elements on one scale can lead to emergent phenomena on another. "Emergence is the reason why there are hurricanes, and ecosystems, and complex organisms like humankind, not to mention traffic congestion and rock concerts. Indeed, the term is positively awe-inspiring" ([44], p. 2). Essentially, this confirms the above contention that 
nature and society not only represent non-linear and dynamic systems, but also coupled socio-ecological, complex systems. It is here, at this juncture, where the theory of complexity takes on practical relevance for risk analysis or hazard management. The concept of behavioral complexity has to be acknowledged and integrated into hazard management for the sake of the future of humanity. The hazard management process is constituted by the entire cyclic process of preparation, emergency and response, reconstruction and mitigation. First and fundamental is the disaster preparation phase which is based on the collective memory of a society and difficult in a context where the last event goes back so long that it is no longer vivid. Social agents themselves do not take part in preparations, and government has to expend a considerable amount of effort every year. The Bahamas example underlines the importance of the socio-cultural context in the development of hazard management concepts. In order to be successful, hazard management needs to be grounded in the way the respective society deals with reality. Although, it will be very difficult to change attitude from searching for linearity to accepting jumps in trajectories, such a change should not only be addressed through early education but in training programs for disaster managers and politicians.

Complexity theory holds that disasters are no exceptions that somehow occur outside the normal course of a system's development. Rather, they form part of a system whose trajectory is characterized by discontinuities and disorder, which should therefore lead us to expect surprise and uncertainty. This strengthens calls for an integrative perspective on disaster and development. Theoretically, this has been known for some time, but practical implementation is still sorely lacking. ${ }^{2}$ Disasters or surprises are part of the normal course of development of a society. A Gestaltwechsel is possible if the course of a system is regarded from the perspective of complexity theory including the notions of surprises and jumps. Interaction of social agents within their regional cultural contexts leads to emergent behavior at the macro level which has to be taken into account in any form of hazard management.

Practical hazard management is a difficult proposition. The example of Haiti shows that in the long run, dangers tend to be ignored if the threat is unspecific. It also shows that much more pressing problems of survival prevent the implementation of locally adapted hazard management strategies. Numerous examples exist for this, demonstrating that disasters are not commonly seen as a constituent part of development. The most recent example of the tropical storm Sandy, which hit New York by surprise, proves this as well. It is a classic mistake not only in developing countries, though their institutions operate slowly and their vulnerability is obvious, direct and immediate economic development tends to be the main focus. Preventative management respecting surprises as part of the system's trajectory seems to hardly exist.

\section{Conclusions}

The theory of complexity explains why integrating community concern in hazard management is more than a democratic imperative. Regional geohazards can be equalled a socially and culturally constructed hazardscape, which requires flexible and adaptive governance structures. There are known

2 Palm [38] was one of the first to present an integrative framework for research and planning for the specific context of natural hazards based on the fundamentals of complexity theory. Possekel [15] applied this approach to the Caribbean island of Montserrat, where she took a multi-scale approach to the situation before and after the volcanic eruption and developed a hazard management concept based on this. 
unknowns. That is to say, there are things that we know we don't know... The above has set out that non-linear dynamic systems are composed of single agent behaviors and interaction at the micro level, which leads to emergence at the macro level. This makes emergence a key parameter in adaptive risk management. Adaptive here means adaptable, flexible and open to feedback, implying the use of a monitoring system to check whether one is still on the desired system trajectory. Adaptive in the context of complexity theory also means taking into account complex agent behavior in their special regional culture, expecting interactions at the micro level to lead to emergence at the macro level.

The example of the Caribbean demonstrates the importance of culture. Working with complexity in hazard research means that agents have to be found, supported and strengthened in the influence they can bring to bear on wider society. Only then can society better prepare for surprises, even where funding continues to be a limiting factor. Surprises are part of the system's trajectory and a constituent part of its future development. Rather than providing justification for doing nothing, this substantiates the need to include a multitude of agents in discussions about future development. Doing nothing may be choosing the worst system trajectory [45].

Recognizing the emergent, historically contingent and self-organizing character of the social world, and developing responsive policy vehicles for managing that complexity, requires a shift in our conception of science in general and of economic science in particular. "We need to embrace more than the simple, and expect less than the fully predictable." ([1], p. 9). New frameworks for policy may be better able to handle the kind of deep uncertainty that social complexity entails. "Adaptive management in place of "predict-and-act" models introduces flexibility to respond to both new situations and new knowledge of the situation." ([1], p. 8) Monitoring and adjusting regulations in light of dynamically changing conditions is a better approach to the kind of complexity found in the social world and preferable to expectations based on the time-honored paradigm of simple, linear, deterministic models. Surprises, ironically, should be expected.

"The most important thing to remember about complexity is very simple:

there's always something yet to come." ([46], p. 46)

\section{References and Notes}

1. Sandra Mitchell, and Wolfgang Streeck. "Complex, Historical, Self-reflexive: Expect the Unexpected!” MPIfG Working Paper 09/15, 2009. Available online: http://www.mpifg.de/pu/workpap/wp09-15.pdf (accessed on 11 November 2012).

2. Beate M.W. Ratter. "Komplexitätstheorie und Geographie-Ein Beitrag zur Begründung einer anderen Sicht auf Systeme.” Mitteilungen der Österreichischen Geographischen Gesellschaft 148 (2006): 109-124.

3. Andreas Schamanek. "Umwelt Management Austria: Einführung in Komplexe Dynamische Systeme.” Unpublished manuscript, 1998. http://www.ams.smc.univie.ac.at/ schamane/kds/ ov.htm (accessed on 25 March 2007).

4. Beate M.W. Ratter. Natur, Kultur und Komplexität: Adaptives Umweltmanagement am Niagara Escarpment Ontario, Kanada. Berlin, Heidelberg: Springer, 2001.

5. Peter Selinger. "Double pendulum." Dalhousie University, 12 May 2000. Available online: http://www.mathstat.dal.ca/ selinger/lagrange/doublependulum.html (accessed on 12 May 2000). 
6. John L. Casti. Complexification-Explaining a Paradoxical World through the Science of Surprise. New York: Harper Perennial, 1994.

7. Danah Zohar, and Ian Marshall. The Quantum Society. New York: William Morrow, 1994.

8. John Urry. "The Complexity Turn." Theory, Culture \& Society 5 (2005): 1-14.

9. Beate M.W. Ratter. "Complexity and emergence - key concepts in non-linear dynamic systems." In Human-Nature Interactions in the Anthropocene: Potentials of Social-Ecological Systems Analysis, edited by Marion Glaser, Gesche Krause, Beate M.W. Ratter, Martin Welp. New York, NY, USA/London: Routledge, 2012, 90-104.

10. Uri Wilensky. "NetLogo Traffic Basic model. Center for Connected Learning and ComputerBased Modeling." Northwestern University, 1997, (accessed on 11 November 2012), http://ccl.northwestern.edu/netlogo/models/TrafficBasic.

11. Roger Lewin. Die Komplexitätstheorie-Wissenschaft nach der Chaosforschung. Hamburg: Hoffmann \& Campe, 1993.

12. Beate M.W. Ratter, and Thomas Treiling. "Komplexität oder was bedeuten die Pfeile zwischen den Kästchen?” In Umwelt als System-System als Umwelt, edited by Heike Egner, Beate M.W. Ratter, Richard Dikau. München: oekom, 2008, 23-37.

13. Steve Rayner. "Cultural Theory and Risk Analysis." In Social Theories of Risk, edited by Sheldon Krimsky, Dominic Golding. Westport: Praeger 1992, 83-115.

14. Christof Tannert, Horst-Dietrich Elvers, and Burkhard Jandrig. "The ethics of uncertainty-In the light of possible dangers, research becomes a moral duty." EMBO Reports 8 (2007): 892-896. doi:10.1038/sj.embor.7401072. PMID 17906667.

15. Anja Possekel. Living with the unexpected-Linking disaster Recovery to Sustainable Development in Montserrat. Berlin, Heidelberg: Springer, 1999.

16. Dennis Lindley. Understanding Uncertainty. Hoboken: John Wiley \& Sons, 2006.

17. Nancy Knight. The Problem of Uncertainty. Institutional Alternatives and Planning Approaches. Vancouver: University of British Colombia, 1991.

18. Kristin S. Shrader-Frechette. "Science versus educated guessing: risk assessment, nuclear waste, and public policy." Bioscience 46 (1996): 488-490.

19. Frank H. Knight. Risk, Uncertainty and Profit. Boston: The Riverside Press, 1921.

20. Bruce Mitchell, ed. Resource and Environmental Management in Canada. Addressing Conflict and Uncertainty. Oxford: Oxford University Press, 1995.

21. Claus-Hennig Hanf. Entscheidungslehre. Einführung in die Informationsbeschaffung, Planung und Entscheidung unter Unsicherheit. München: Oldenbourg, 1986.

22. Brian Arthur. Increasing Returns and Path Dependence in the Economy. Ann Arbor: University of Michigan Press, 1994.

23. Ortwin Renn. "Concepts of Risk: An interdisciplinary review. Part 1 Disciplinary Risk Concepts." GAIA 17 (2008): 50-66.

24. Peter L. Berger, and Thomas Luckmann. Die gesellschaftliche Konstruktion der Wirklichkeit. Eine Theorie der Wissenssoziologie. Frankfurt/Main: Fischer Taschenbuch Verlag, 1987.

25. Janet Stephenson. "The Cultural Values Model: An integrated approach to values in landscapes." Landscape and Urban Planning 84 (2008): 127-39. 
26. Kira Gee, and Benjamin Burkhard. "Cultural ecosystem services in the context of offshore wind farming: A case study from the west coast of Schleswig-Holstein." Ecological Complexity 7 (2010): 349-358. doi:10.1016/j.ecocom.2010.02.008.

27. Mark W. Corson. "Hazardscape in reunified Germany." Environmental Hazards 1 (1999): 57-68.

28. Susan L. Cutter, Jerry T. Mitchell, and Michael S. Scott. "Revealing the vulnerability of people and places: A case study of Georgetown County, South Carolina." Annals of the Association of American Geographers 90 (2000): 713-37. doi: 10.1111/0004-5608.00219.

29. Shabana Khan, and Michael J. Crozier. "Hazardscape-A holistic approach to assess tipping points in humanitarian crisis." UNU-MRF Summer Academy on Social Vulnerability, 2009. www.ehs.unu.edu/file/get/4127 (accessed on 11 November 2012).

30. Jeff Malpas, and Gary Wickham. "Governance and Failure: On the Limits of Sociology." Australian and New Zealand Journal of Sociology 31 (1995): 37-50.

31. US Geological Survey. "Earthquake Hazard and Safety in Haiti and the Caribbean Region." 21 January 2010. www.supersites.unavco.org/Haiti_triggering.pdf (accessed on 11 November 2012).

32. Paul Mann, Eric Calais, Chuck Demets, Carol Prentice, and Margaret Wiggins-Grandison. "Enriquillo-Plantain Garden Strike-Slip Fault Zone: A Major Seismic Hazard Affecting Dominican Republic, Haiti And Jamaica." Paper presented at 18th Caribbean Geological Conference, Austin, March 24-29, 2008, www.ugr.es/ agcasco/igcp546/DomRep08/Abstracts_ CaribConf_DR_2008.pdf (accessed on 11 November 2012).

33. Pamela E. Jansma, and Glen S. Mattioli. "GPS results from Puerto Rico and the Virgin Islands: Constraints on tectonic setting and rates of active faulting" Geological Society of America Special Papers 385 (ed. Paul Mann) (2005): 13-30.

34. Department of Meteorology, The Bahamas. "Tropical storms and hurricanes affecting The Bahamas." Unpublished manuscript, 2008. see also www.bahamasweather.org.bs/.

35. Luise K. Comfort, ed. Managing Disaster. Strategies and Policies Perspectives. London: Duke University Press, 1988.

36. Bruce Mitchell. Geography and Resource Analysis. Essex: Longman Scientific \& Technical, 1989.

37. Bruce Mitchell. Resource and Environmental Management. Harlow: Longman, 1997.

38. Risa I. Palm. Natural Hazards. An Integrative Framework for Research and Planning. Baltimore: Johns Hopkins University Press, 1990.

39. Jan Vermeiren. "Natural disasters. Linking economics and environment with a vengeance." In Caribbean Ecology and Economics, edited by Norman P. Girvan, David A. Simmons. Barbados: Caribbean Conservation Association, 1991, 127-42.

40. Stephen R. Dovers, and John W. Handmer. "Uncertainty, sustainability and change." Global Environmental Change 2 (1992): 262-76.

41. Mariana Mossler. "Environmental hazard analysis and small island states. Rethinking academic approaches." Small islands, large questions. Special issue Geograpische Zeitschrift 84 (1996): 86-93.

42. Andreas Klinke, and Ortwin Renn. "Systemic Risks as Challenges for Policy Making in Risk Governance." Forum Qualitative Social Research 1 (2006): Art. 33. www.qualitativeresearch.net/index.php/fqs/article/view/64/132 (accessed on 11 November 2012). 
43. Ortwin Renn. "Concepts of Risk: An interdisciplinary review. Part 2 Integrative Approaches." GAIA 2 (2008): 196-204.

44. Peter A. Corning. "The Re-Emergence of 'Emergence': A venerable concept in search of a theory." Complexity 7 (2002): 18-30.

45. Klaus Mainzer. Thinking in Complexity. Berlin: Springer, 1997.

46. Wolf Lotter. "Einfach mehr." brand eins 8 (2006): 46-55.

(C) 2013 by the authors; licensee MDPI, Basel, Switzerland. This article is an open access article distributed under the terms and conditions of the Creative Commons Attribution license (http://creativecommons.org/licenses/by/3.0/). 